\title{
Jacques Noiray, Le Simple et l'Intense. Vingt études sur Émile Zola
}

\section{Maria Emanuela Raffi}

\section{(2) OpenEdition}

1 Journals

\section{Edizione digitale}

URL: http://journals.openedition.org/studifrancesi/5408

DOI: $10.4000 /$ studifrancesi. 5408

ISSN: 2421-5856

\section{Editore}

Rosenberg \& Sellier

\section{Edizione cartacea}

Data di pubblicazione: 1 dicembre 2016

Paginazione: 556-558

ISSN: 0039-2944

\section{Notizia bibliografica digitale}

Maria Emanuela Raffi, « Jacques Noiray, Le Simple et l'Intense. Vingt études sur Émile Zola », Studi

Francesi [Online], 180 (LX | III) | 2016, online dal 01 janvier 2017, consultato il 18 septembre 2020.

URL : http://journals.openedition.org/studifrancesi/5408; DOI : https://doi.org/10.4000/studifrancesi. 5408

Questo documento è stato generato automaticamente il 18 settembre 2020.

\section{(c) 9 (i) $\Theta$}

Studi Francesi è distribuita con Licenza Creative Commons Attribuzione - Non commerciale - Non opere derivate 4.0 Internazionale. 


\title{
Jacques Noiray, Le Simple et l'Intense. Vingt études sur Émile Zola
}

\author{
Maria Emanuela Raffi
}

\section{NOTIZIA}

JACQUES NOIRAY, Le Simple et l'Intense. Vingt études sur Émile Zola, Paris, Classiques Garnier, 2015, $381 \mathrm{pp}$.

1 Jacques Noiray aggiunge con questo volume su Zola un ulteriore prezioso contributo ai suoi numerosi studi sul romanzo realista e naturalista. Vi sono raccolti diciotto articoli pubblicati in varie sedi - alcune non facilmente reperibili e di cui si renderà dunque conto - fra il 1993 e il 2015 e due scritti inediti, tutti riuniti sotto un titolo ispirato a un'affermazione dello stesso Zola - «Avoir l'impression forte de ce dont on parle, et rendre cette impression avec la plus grande intensité et la plus grande simplicité, c'est l'art d'écrire tout entier»-e che per Noiray struttura e definisce il vasto insieme della sua opera.

2 Nello studio introduttivo al volume (Zola, le simple et l'intense, inedito), Noiray mostra la presenza delle due caratteristiche nell'opera zoliana. La volontà di semplificazione dettata dal naturalismo, che porta a un intreccio fondato su documenti e a personaggi costruiti seguendo biografie prese dalla realtà, appare infatti utilizzata per un obiettivo più ambizioso: “"faire de la vie" avec les éléments que ce réel lui fournit», grazie a una «expression personnelle» che rende unica la sua opera narrativa. La «quête d'intensité» appare quindi ambiguamente già all'interno della semplificazione zoliana e assume un peso sempre più importante nella sua opera. Qualificata spesso da Zola come una sorta di patologia dell'epoca attraverso la parola «hypertrophie», «l'expression intense de la réalité» mostra stretti legami con la rappresentazione teatrale e si realizza attraverso una «rhétorique individuelle», dettagliatamente esaminata da Noiray, il cui fine ultimo appare come un ideale totalizzante: «l'union intime et passionnée de l'homme et de la totalité du monde». 

nostalgico di giovinezza, libertà e comune amore per la Provenza, ma la cui superiorità appare evocata soprattutto per smentire e allontanare eventuali legami con Victor Hugo e respingere «une relation de dépendance ambiguë mais féconde avec un modèle hugolien dont l'influence, même niée, ne cesse de se faire sentir». Molto più concreto appare naturalmente il legame con Flaubert ("Pot-Bouille", ou "L'Éducation sentimentale" d'Emile Zola, 2000), di cui Noiray analizza qui lo stretto rapporto di filiazione di PotBouille dall'Éducation sentimentale, non privo di qualche méprise interpretativa da parte di Zola e pervaso comunque dalla presenza della grande ombra hugoliana.

5 In «Formes» sono raccolti quattro contributi. In Figures du grotesque dans "La Fortune des Rougon" (2015), Noiray riprende la nozione di grottesco elaborata da Hugo, la cui caratteristica «c'est d'être visible et d'outrer autant que possible cette visibilité» e ne studia la presenza nei Rougon-Macquart e soprattutto in La fortune des Rougon, primo romanzo del ciclo, in cui il grottesco appare quasi sottolineato e declinato in diversi contesti («Le grotesque dans l'histoire», «Le grotesque au cœur du tragique»).

6 Saccard, personnage reparaissant (2002), propone un'analisi ampia e dettagliata del personaggio di Aristide Saccard, presente in ben quattro romanzi zoliani ed espressione privilegiata di quella «épopée matérialiste du désir et de la jouissance», costituita dall'insieme dei Rougon-Macquart. «Ce qu'il incarne-scrive Noiray-c'est le grand mouvement qui conduit l'œuvre de Zola de son pessimisme initial [...] à une célébration ambiguë, puis nettement déclarée, des forces de vie».

7 L'interesse dello studioso si sposta agli ultimi romanzi di Zola in Aspects de l'ironie dans "Paris" (2002); «cycle de la souffrance et de la pitié», Les Trois Villes rivelano tuttavia per Noiray - e Paris in particolare - una costante ironia che si esprime sia in una galleria di personaggi grotteschi, sia in attacchi più generali all'immoralità della società mondana e del Parlamento, accompagnati dalle critiche al dandysmo fin-de-siècle, all'idealismo simbolista, alle ingenuità del socialismo e della carità impotente. La conclusione del romanzo e i suoi due ultimi libri appaiono tuttavia più lucidi che ironici: lo sguardo dell'autore si fa più grave e all'ironia subentra il sogno dell'utopia.

Per "Fécondité", conte, légende, féerie si rinvia all'ultima parte della scheda di "Studi francesi» (n. 175, pp.177-180) sul volume Le Réalisme et ses paradoxes (1850-1900) in cui l'articolo è stato pubblicato nel 2014.

9 La sezione «Idées» si apre con "Germinal", roman mystique (inedito), in cui Noiray considera le importanti «interférences du social et du religieux» che fanno procedere il romanzo verso un'attesa sociale di tipo mistico, che dovrebbe realizzare subito il paradiso in terra e raddrizzare ogni tipo di sopruso. La diffidenza di Zola nei confronti di questo tipo di socialismo, che pure alimenta e fa crescere nel testo di Germinal, appare attraverso «une double série de métaphores opposées» relative rispettivamente alle tenebre della disperazione e dell'abbandono e alla "germination», movimento verso la luce. Fedele ai princìpi del naturalismo, Zola sembra osservare il fenomeno con distaccata neutralità, ma le conclusioni verso cui il romanzo sospinge il lettore sembrano chiare a Noiray: poiché «le socialisme n'est pas une solution à la question sociale [car] il se glisse trop facilement dans les habits de la vieille religion caduque», 
«la science sera, dans l'ordre et dans la raison, la seule ouvrière efficace du progrès». Salvo a reincontrare sulla sua strada, negli ultimi romanzi, il difficile scoglio del «besoin de croire».

Médecine et miracle dans "Lourdes" (2000) prende in esame il secondo romanzo dedicato da Zola alla medicina, dopo Le Docteur Pascal, questa volta attraverso la singolare prospettiva del miracolo, in cui si incontrano medicina e religione. Lourdes appare come terra di elezione per un simile incontro, caratterizzata com'è dal «renversement des lois de la nature et de la religion», ma la scienza riesce a trovarvi comunque le sue ragioni di ordine psichico-soprattutto l'autosuggestione sia individuale che collettiva - non ancora del tutto conosciute. È dunque a una nuova medicina, ispirata a Charcot e che "garde une part de mystère, parce qu'elle réserve une place au rêve et à la fiction» che Zola affida in Lourdes la spiegazione del "miracolo".

11 In Zola devant la décadence (2012) Noiray indaga i rapporti che Zola intrattiene con la sensibilità decadente, a partire dalla visione catastrofica della natura umana e della società contemporanea, che si esprime nei suoi romanzi attraverso l'unione ossimorica di due elementi principali: «l'épuisement physique et la surexcitation nerveuse». Questa visione del mondo si riverbera anche sull'immagine dello scrittore e anzitutto su quella dei Goncourt la cui opera viene definita da Zola nel 1865 «fiévreuse et maladive», oltre che «moderne» e «bizarre»; l'ammirazione per la scrittura «artiste» si mescola alla diffidenza che, a partire dal 1870, si fa apertamente critica: «"être une force" deviendra bientôt, contre toutes les tentations du pessimisme ou de l'asthénie morbide, le mot d'ordre de son œuvre et même de sa vie».

12 La quarta parte "Thèmes» si apre con Le paysage dans "Rome" (2004), già recensito in questi «Studi» come parte del volume collettivo Actualité de Zola en l'an 2000. La presenza della scultura nell'opera di Zola è l'argomento dell'articolo successivo (À la recherche de la sculpture naturaliste, 2012), in cui Noiray considera i numerosi scultori che popolano i Rougon-Macquart esseri forti, popolari e vagamente primitivi, spesso tentati tuttavia dal «joli» e dalle figurine di genere cui Zola sembra concedere via via sempre più credito. La scultura appare in particolare sempre connessa al corpo femminile e i personaggi femminili zoliani, spesso presentati in pose statuarie, sembrano riunire diverse istanze legate, naturalisticamente, alla natura e alla vita: «beauté charnelle de la femme, beauté spectaculaire de la représentation théâtrale, beautéplastique de la statue». Quand Zola revisite la Bible (2011) fa il punto sulla "culture biblique» dello scrittore naturalista, cultura marginale, espressa da residui e frammenti, con poche citazioni puntuali e una presenza molto più significativa a livello immaginario. $\grave{E}$ particolarmente il mito di Adamo ed Eva a percorrere l'opera zoliana, da La Faute de l'abbé Mouret a Le Docteur Pascal e infine a Paris, attraverso una progressiva trasformazione e paganizzazione: «soulagé de ses implications érotiques, délivré de toute idée religieuse ou morale de faute et de châtiment, le mythe d'Adam et Ève, édulcoré et simplifié, aboutit dans Paris à ce qui est à la fois sa perfection et sa fin».

Nella quinta parte-«Images» -, Noiray prende in esame anzitutto un'immagine ricorrente negli ultimi romanzi zoliani: il «fleuve de lait» (De la catastrophe à l'apaisement, 1993). Specificazione biblica dei molti «ruissellements» presenti nell'opera precedente, discendenza rigeneratrice del «fleuve de boue» e del successivo «fleuve de larmes», la metafora del fiume di latte si moltiplica nelle ultime opere «en même temps que se développe dans les Villes et les Évangiles une triple tendance au sentiment, à la moralisation et à 1 abstraction». Sur deux instruments symboliques du naturalisme (2004) 
individua nel bisturi e nel trampolino gli strumenti simbolici del romanziere naturalista, produttore di uno studio di anatomia umana e al tempo stesso capace di elevarsi vertiginosamente al di sopra di essa. Come mostra Noiray, Zola raccoglie la doppia eredità di Flaubert (e prima di Balzac) et di Banville (e prima di Hugo) combinandole nella sua visione della creazione naturalista: «le rôle de l'écrivain, à côté du savant et même en avant de lui, sera d'“aller du connu à l'inconnu", de formuler des hypothèses que la science se chargera de vérifier». L'azione della politica nel romanzo zoliano è ciò che appare al centro dello studio successivo - L'imaginaire de la politique dans Paris (2000) -, in cui Jacques Noiray si interrroga anzitutto sul concetto di «roman politique» per poi concentrarsi su Paris, romanzo che mette in scena il caos della vita parlamentare, la vanità delle ideologie politiche e su tutto ciò la nocività di una stampa corrotta e menzognera per poi risollevare tutto in una visione che Jaurès definirà «"le quiétisme scientifique" de Zola».

La sesta e ultima parte, «Obsessions», presenta anzitutto uno studio su un elemento fondamentale, la genesi di un romanzo zoliano, nel caso specifico Rome (De la mise à nu comme principe poétique, 2002), da un fantasma dell'immaginario: «le fantasme de la femme qui se désahabille». Inizialmente dedicato al tema del «socialisme catholique», già nel 1894 il progetto, come osserva Noiray, «sur les 44 feuillets qui composent la première ébauche, 19 sont consacrées à la préparation détaillée de la grande scène de la mort des amants». Da tale scena, fissata per prima da Zola, viene, oltre a tutta la struttura del romanzo, anche l'immagine centrale di Benedetta, vergine che sacrifica per amore il proprio pudore di fronte all'innamorato morente, spogliandosi in una lunga scena che prelude alla morte di entrambi gli amanti. In Zola romancier de la force (2006) continua l'indagine di Noiray sugli «elementi fisici» dell'opera di Zola, che manifesta in più occasioni un'attrazione per la forza, a partire dal massiccio Eugène Rougon, per continuare con l'abbé Faujas (La Conquête de Plassans) e con Nantas (protagonista eponimo di una novella). In tutti e tre «le culte de la force aboutit bien à une mystique de la volonté» che diventa in un primo momento pericolosa e inquietante per poi rappresentare sempre più nell'opera zoliana il segno distintivo del grande autore e dell'eroe forte e positivo, cui si aggiunge una connotazione estetica: «la force physique et morale s'épanouit et se sublime en bonté [...] la forme visible de la force, l'enveloppe de la bonté, c'est la beauté». Zola, mémoire et vérité de la chair (2008) affronta il tema della «sensation» nell'opera di Zola. Per Noiray le sensazioni zoliane sono sempre fissate nella loro «substance charnelle» ed è dunque l'uso del termine chair, molto frequente, a essere particolarmente analizzato, dato che esso sembra rinviare non solo alla carnalità riconosciuta dell'opera narrativa, ma anche a una sorta di segreto profondo che nella chair va cercato e svelato. Poco prima dell'avvento di Freud, Zola s'interroga sul mistero del rapporto fra corpo, memoria e sensazione: «Quelle est cette trace en nous, cette marque imprimée dans la chair depuis l'origine des temps?». La raccolta si chiude con De La Confession de Claude à «'accuse» (2004) in cui Noiray studia una delle «formes obsédantes» dell'immaginario zoliano - la figura del sacrificio -, esaminata in diversi romanzi, da La confession de Claude a La Curée, a Le Ventre de Paris, fino a Les Trois Villes, passando anche attraverso molti altri testi narrativi. Definito come «la forme di religieux dramatisé», il tema del sacrificio permea di sé anche la partecipazione di Zola al caso Dreyfus e in particolare «J'accuse», in cui il romanziere ritrova l'immagine hugoliana «du héros solitaire, en lutte pour le bonheur de tous contre un pouvoir injuste et méchant», eroe che offre se stesso in sacrificio per fondare una società futura più giusta. 